\title{
Extraintestinal Manifestations of Inflammatory Bowel Disease
}

\author{
Folashade A. Jose ${ }^{\star}$ and Melvin B. Heyman ${ }^{\dagger}$ \\ *Department of Pediatric Gastroenterology, Hepatology, and Nutrition, University of California, \\ San Francisco \\ †Department of Pediatrics, University of California, San Francisco
}

\section{Keywords}

Colitis; Crohn disease; Erythema nodosum; Growth; Pediatric; Sclerosing cholangitis; Thromboses

\begin{abstract}
Extraintestinal manifestations (EIMs) of inflammatory bowel disease (IBD) have been reported to involve almost every organ system. This article focuses on the prevalence (where known) and clinical aspects of the more commonly described EIMs of Crohn disease (CD) and ulcerative colitis (UC). Because of limited data from pediatric populations, much of the information in this review is from adult reports. An important consideration is whether EIMs represent manifestations of the same pathogenetic mechanisms or distinctly different disease processes from the underlying IBD, possibly coincidental to the presence of IBD or as a consequence of the IBD or its treatment. EIMs may present before the onset of gastrointestinal symptoms, during the natural course of disease, and rarely may even follow colectomy in patients with UC.
\end{abstract}

The prevalence of at least 1 EIM in recent population-based studies of patients with IBD ranges from $6 \%$ to $47 \%$ (1-4). Several reports from pediatric centers suggest a greater prevalence in younger patients. Grossman and DeBenedetti (5) reported that $68 \%$ of 41 children with IBD had EIMs, and a recent review of pediatric patients by Stawarski et al (6) reported that $50 \%$ with $\mathrm{UC}$ and $80 \%$ with $\mathrm{CD}$ had at least 1 EIM.

In this review, we provide an overview of EIMs that have been reported to be associated with UC and CD, emphasizing the pediatric experience when such information is available. EIMs are subdivided into several categories: colitis related, specifically skin, eye, joint, and mouth, where the activity of the EIM parallels the activity of the underlying intestinal disease; hepatobiliary; impaired growth; EIMs secondary to complications of or as direct extensions of bowel disease, more frequently noted in patients with $\mathrm{CD}$ than with $\mathrm{UC}$ and including nephrolithiasis, obstructive uropathy, cholelithiasis, and pancreatitis; and EIMs that cannot be categorized clearly in one of the other groups, such as amyloidosis and cancer (7). EIMs involving vascular, hematological, pulmonary, cardiac, and neurological systems probably belong to this last group. Impaired growth, unique to the pediatric age group, may be an EIM potentially related to all of these categories. Iatrogenic EIMs, such as druginduced bone marrow suppression and corticosteroid-associated myopathy, are not discussed (Table 1).

(C) 2008 by European Society for Pediatric Gastroenterology, Hepatology, and Nutrition and North American Society for Pediatric Gastroenterology, Hepatology, and Nutrition

Address correspondence and reprint requests to Melvin B. Heyman, MD, MPH, Anita Ow Wing Endowed Chair, Professor of Pediatrics, 500 Parnassus Ave, MU 4-East, Box 0136, University of California, San Francisco, CA 94143-0136 (mheyman@peds.ucsf.edu). 


\section{PATHOGENESIS}

The pathogenesis of EIMs associated with IBD is poorly understood. Many EIMs are hypothesized to be due to immune reactions, supported by the observations that primarily immunological derangements progress to the development of IBD and that patients with IBD have an increased risk of autoimmune diseases (8). Malabsorption, protein-losing enteropathy, fistulas, and intestinal resection each can result in deficiencies of nutrients, proteins, bile salts, vitamins, and minerals with corresponding clinical consequences. Medications used in the treatment of IBD may be associated with many nonintestinal as well as intestinal side effects.

Animal studies support the theory that intestinal bacteria also play a pathological role in the development of EIMs. In HLA-B27 transgenic and T cell receptor- $\alpha$ mutant colitis mouse models, the expression of extraintestinal (and intestinal) disease requires the presence of commensal bacteria; these mouse models do not manifest intrinsic intestinal or extraintestinal pathologies in germ-free environments (9).

Genetic factors also have been implicated in the pathogenesis of EIMs in IBD. Ethnic and racial differences in IBD phenotype have potential implications for diagnosis and management of IBD and its complications (10). Studies reveal associations of EIMs in IBD with major histocompatibility complex loci. For examples, patients with $\mathrm{CD}$ who have EIMs are more likely to have HLA-A2, HLA-DR1, and HLA-DQw5, whereas patients with UC and EIMs are more likely to have HLA-DR103, B27, and B58 phenotypes $(11,12)$.

\section{COLITIS-RELATED EXTRAINTESTINAL MANIFESTATIONS}

\section{Dermatological Manifestations}

Skin involvement has been described in $10 \%$ to $15 \%$ of patients with IBD (7). The 2 most commonly encountered cutaneous manifestations of IBD are erythema nodosum and pyoderma gangrenosum. Erythema nodosum presents as a single or multiple tender red nodules typically on the extensor surface of the lower extremities. Reported to be more common in CD than UC, erythema nodosum correlates well with and usually reflects increasing bowel activity $(1,5,7)$. Bernstein et al (1) reviewed EIMs in 4445 adult patients with IBD and found prevalence rates of erythema nodosum of $1.9 \%$ in patients with $\mathrm{CD}$ and UC. A report based on 41 children with IBD found 4 of 32 (12.5\%) children with UC and 5 of $9(56 \%)$ with CD had erythema nodosum (5). Several other reports, again, all in adults, report prevalence rates of $2 \%$ to $7.5 \%$ in $\mathrm{CD}$ and $0.9 \%$ to $4 \%$ for $\mathrm{UC}(2,7,13-15)$.

The etiology of erythema nodosum is unknown, although a distinct HLA region on chromosome 6 (HLA-B15) is weakly associated with its development, supporting some genetic influence (12). These lesions usually respond to treatment of the underlying IBD. Although some success has been reported with oral potassium iodide, severe or refractory cases may require systemic corticosteroids or immunosuppressive therapy.

Pyoderma gangrenosum usually begins as an erythematous pustule or nodule that spreads rapidly to adjacent skin and develops into a burrowing ulcer with irregular violaceous edges. Although the appearance can be dramatic, the ulcers are sterile. Lesions are found typically on the extensor surfaces of the extremities, but may appear in other portions of the body, especially sites of trauma. Pyoderma gangrenosum has been reported to evolve from preceding erythema nodosum (16). Pyoderma gangrenosum is reported in $0.5 \%$ to $5 \%$ of patients with IBD $(2,7,15)$. Most reports suggest that it is more common in patients with UC than those with CD, however, in the study by Bernstein et al (1) of 4445 patients a higher 
prevalence was found among patients with $\mathrm{CD}$. Specific prevalence data in children are lacking.

Four distinctive variants of pyoderma gangrenosum are recognized: bullous, familial, malignant, and superficial granulomatous pyoderma. Pyoderma gangrenosum may develop before bowel symptoms, during quiescent disease, or even following colectomy. About $40 \%$ of IBD patients with pyoderma gangrenosum develop arthritis (14). Lesions are multiple in the majority of patients, and most appear below the knee. Histological characteristics include lymphocytic vasculitis peripherally in the erythematous zone, neutrophilic infiltration, and a central abscess. Direct immunofluorescence may show immunoglobulin and complement deposits involving superficial and deeper dermal vessels.

Pyoderma gangrenosum often resolves with treatment of the underlying IBD. Mild cases usually respond to local and topical therapy, including intralesional corticosteroid, topical cromolyn sodium, and topical 5-aminosalicylic acid. Effective systemic agents include oral sulfasalazine, dapsone, corticosteroid, and immunomodulators such as azathioprine, cyclophosphamide, cyclosporine, methotrexate, tacrolimus, and mycophenolate mofetil (17). Successful treatment with infliximab in both children and adults has been documented $(18,19)$.

Metastatic $\mathrm{CD}$ is a granulomatous dermatitis seen in adults, although isolated cases have been described in children(20). Acute febrileneutrophilic dermatosis (Sweet syndrome) is a rare EIM of IBD and presents as tender, erythematous plaques or nodules involving the arms, legs, trunk, hands, or face (21). It may parallel the intestinal disease or may precede the diagnosis of IBD. Skin biopsies reveal neutrophilic infiltrate with leukocytosis. Azathioprine has been implicated as a possible cause of Sweet syndrome in a patient with CD. Most cases respond to systemic corticosteroids, and metronidazole is reported to be effective in 1 case (22-24). Other cutaneous manifestations associated with IBD include psoriasis, epidermolysis bullosa, polyarteritis nodosa, alopecia, and erythema elevatum diutinum $(25,26)$.

\section{Ophthalmological Manifestations}

Ophthalmologic involvement related to IBD is reported in $1.6 \%$ to $4.6 \%$ of patients with UC and $3 \%$ to $6.3 \%$ of patients with $\mathrm{CD}(2,4,15,27,28)$. Patients with CD with colonic involvement are more likely to develop ocular problems than those with small bowel disease alone. Data suggest that asymptomatic transient uveitis is common in children with granulomatous bowel disease, but progression to severe adult uveal disease is poorly documented. Ophthalmological changes also may result from treatment rather than directly from the underlying IBD. Involvement of the vascular coats of the eye in IBD may be from bacteria or genetic and immunological factors in the manifestation of extraintestinal symptoms of IBD $(29,30)$.

The most common ocular lesions associated with IBD are episcleritis and uveitis. Episcleritis is characterized by painless hyperemia of the sclera and conjunctiva without loss of vision. Uveitis manifests as an acute or subacute painful eye with visual blurring, photophobia, headache, and iridospasm. Visual acuity is not affected unless the retina or the posterior uveal structures are involved. Diagnosis is made on slit lamp examination, showing flare and cells in the anterior chamber. Acute anterior uveitis is an ophthalmological emergency requiring prompt intervention. Prompt treatment of uveitis with systemic or topical steroids is essential to prevent progression to blindness. Episcleritis rarely progress to blindness if untreated. 
Ocular complications also may develop in patients with IBD due to chronic corticosteroid usage. Increased intra-ocular pressures are reported in $22 \%$ of pediatric patients exposed to varying doses of corticosteroids (31). The risk of corticosteroid-induced glaucoma leading to irreversible visual loss and corticosteroid-associated cataract should prompt careful ophthalmological evaluation at regular intervals in patients with chronic corticosteroid use. Recent reports of glaucoma also have been noted among children not exposed to corticosteroids, again suggesting an immunological mechanism for its development.

\section{Musculoskeletal Manifestations}

The most common EIMs in IBD are musculoskeletal disorders. Spondyloarthropathy is reported in $5 \%$ to $20 \%$ of patients with $\operatorname{IBD}(7,15,27,28,32)$. The 2 patterns described in both children and adults are a peripheral arthritis, sometimes referred to as "colitis arthritis," and the axial form, "ankylosing spondylitis" or "sacroiliitis" (33). The peripheral arthropathy associated with IBD has been subclassified into 2 distinct types. Type 1 is pauciarticular arthritis. It affects fewer than 5 joints, is asymmetrical, and typically affects large joints (knee, elbow, and ankles). It usually manifests with acute self-limiting episodes lasting a median of 5 weeks. Approximately $20 \%$ to $40 \%$ of all patients have more than 1 episode of arthritis. In one of the few early reports in children $(n=41), 5$ of $32(16 \%)$ with UC and 2 of 9 (22\%) with CD had arthritis (5). The risk of developing peripheral arthropathy appears to increase with the extent of colonic disease. It also is increased in association with disease complications or other EIMs including abscesses, perianal disease, erythema nodosum, stomatitis, uveitis, and pyoderma gangrenosum. In contrast, type 2 peripheral arthropathy is polyarticular, involving 5 or more joints, more typically the small joints. Polyarticular arthritis with IBD is independent of intestinal disease activity but has been associated with uveitis (12). Symptoms of polyarticular arthritis are reported to persist for a median of 3 years. The development of articular manifestations in patients with UC appears to be influenced by genetic factors present in some major histocompatibility complex haplotypes (34). Type 1 peripheral arthritis is associated with HLA-B27, HLAB35, and HLA-DR, whereas type 2 is associated with HLA-B44.

Axial arthropathy, both spondylitis and isolated sacroiliitis, is less frequent than peripheral arthropathy and is reported in $3 \%$ to $25 \%$ of patients $(7,15,27,28)$. As with polyarticular disease, axial arthropathy does not parallel IBD disease activity. Ankylosing spondylitis is reported in 5\% to 10\% of patients with IBD; most are young (adolescents and young adults) and most are HLA-B27 positive. Ankylosing spondylitis in the HLA-B27-positive patients has a disease course that is identical to ankylosing spondylitis seen in the spondyloarthropathies. Patients with ankylosing spondylitis often experience severe onset of back pain associated with morning stiffness or exacerbated by periods of rest. Symptoms usually are unrelated to the underlying IBD disease activity. Radiographs in early stages may be normal or show only minimal sclerosis.

Peripheral arthropathy usually responds to medical and surgical treatment of the underlying colitis. Other treatment modalities include rest, physical therapy, and intraarticular steroid injections. Nonsteroidal anti-inflammatory drugs used in the management of IBD-associated peripheral arthropathy require caution because of the reported association of exacerbation of IBD and their use, although ongoing studies will provide updated information regarding this controversial issue (35). In contrast to peripheral arthropathy, which usually responds to treatment of colitis, medical or surgical therapy of the underlying IBD does not alter the progressive nature of the axial arthropathy. Other medical therapies have included sulfasalazine, mesalamine, methotrexate, azathioprine, and anti-tumor necrosis factor therapy with infliximab or thalidomide (36). 


\section{Osteoporosis/Osteopenia}

Osteoporosis and osteopenia have been reported in $23 \%$ to $59 \%$ of adult patients with IBD $(37,38)$. Osteopenia was observed in $41 \%$ of children with CD and in $25 \%$ with UC (6). In a study of 42 children, bone-mineral disturbances were found in $57 \%$ of cases, with predominance of osteopenia among patients with UC (24\%) and osteoporosis among those with CD (24\%) (39). In another study of 90 children (50 girls) with IBD (34 free of steroids, 53 treated with steroids, and 3 with unknown usage) and 50 controls ( 27 girls), the rate of osteoporosis was $8 \%$ in girls and $20 \%$ in boys, similar to adult reports. A similar proportion of osteoporosis is reported in non-steroid-treated (12\%) and steroid-treated (11\%) patients (40).

Osteoporosis is a systemic skeletal disease characterized by low bone mass and microarchitectural deterioration leading to increased bone fragility and susceptibility to fracture. Bone density can be estimated using bone densitometry (eg, dual energy x-ray absorptiometry). The densimetric criteria for bone loss are based on $t$ and $z$ scores, which are standard-deviation scores expressed in relation to reference values in young healthy subjects $(t$ score) or sex- and age-matched healthy controls ( $z$ score). Osteopenia is defined as a score of -1 to -2.5 , and osteoporosis is a score lower than -2.5 .

The pathogenesis of osteopenia or osteoporosis in IBD is not well understood. Several factors may interfere with bone promotion and accelerate bone destruction in patients with IBD. Important potential pathogenetic factors in IBD include hypogonadism induced by IBD, manifestation with absence of menses in women, malabsorption of calcium and/or vitamin D, low body mass index, corticosteroid exposure, and disease activity and related elevation of inflammatory cytokines (41). Patients with CD may develop osteoporosis or osteopenia even without treatment with corticosteroids.

Patients with CD should undergo baseline bone densitometry scans, with follow-up imaging studies variably recommended 1 to several years later. With a family history of osteoporosis, bone densitometry should be done sooner and more often. Bone densitometry has been recommended at 1- to 2-year intervals for pediatric patients with long-term, high-dose exposure to corticosteroids, although even adult patients with corticosteroid treatment equivalent to a relatively low dose of $7.5 \mathrm{mg}$ of prednisone for 6 months' duration also may develop significant bone loss and should be monitored carefully (42). Special attention should be directed to ensuring that patients taking corticosteroids have adequate calcium and vitamin D intakes. Prevention of bone loss should begin with an attempt to limit corticosteroid-induced bone loss by minimizing the corticosteroid dosage, substituting with steroid-sparing immunomodulators or adding an agent to enhance bone-mineral uptake. Bisphosphonates and calcitonin have been applied in patients with IBD for the treatment of osteoporosis, although their application in pediatric patients remains to be demonstrated (43).

Osteonecrosis (also referred to as aseptic necrosis or avascular necrosis) is characterized by death of osteocytes, adipocytes, and hematopoietic cells in the bone and eventual bone collapse. It has been described in both children and adults with IBD, with a reported prevalence in 1 series of $<1 \%$ of all patients and up to $4 \%$ of patients taking corticosteroids (44). Early recognition of this potentially serious EIM is important for successful outcome. Diagnosis is made by bone scan and magnetic resonance imaging. Treatment may include medical management (eg, calcium, vitamin D, hydrochlorothiazide, bisphosphonates). Surgical therapies such as core-decompression biopsy and unicompartmental arthroplasty also have been recommended (45). 


\section{Oral Manifestations}

Oral aphthous stomatitis is seen in at least 5\% to $10 \%$ of patients with UC and $20 \%$ to $30 \%$ with CD. Oral lesions may occur before the onset of intestinal symptoms of IBD or may parallel intestinal disease activity. Symptoms include mild discomfort to severe debilitating pain. Biopsies of these lesions often reveal lymphedema and, in patients with CD, granulomata. Treatment with systemic or local corticosteroids or other immunosuppressive medications has been effective.

Pyostomatitis vegetans is a rare, benign, chronic disease characterized by miliary pustules, erosions, and vegetating dermatosis of the skin and oral mucosa. Oral lesions are distinct and appear as multiple pustules with an erythematous base that coalesce and undergo necrosis to form a typical "snail tracks" appearance. Pyostomatitis vegetans has a strong association with gastrointestinal disease and is considered a marker for underlying IBD; most reported cases have been associated with UC. Pyostomatitis vegetans also has been associated with zinc deficiency in a patient with underlying oral CD (46). Management is based on treating the underlying gastrointestinal disease using dietary modification and systemic agents such as antibiotics, sulfasalazine, corticosteroids, azathioprine, and dapsone. Surgical treatment in severe cases of UC involves total colectomy and has resulted in permanent remission of the pyostomatitis vegetans (47).

\section{HEPATOBILIARY MANIFESTATIONS}

Hepatobiliary complications are among the more serious EIMs in patients with either UC or $\mathrm{CD}$, and include small bile-duct and large bile-duct inflammation, hepatitis (chronic active, drug induced, granulomatous), cirrhosis, bile-duct carcinoma, fatty liver, amyloidosis, hepatic abscess, and cholelithiasis. The most common serious hepatobiliary complication among pediatric patients is primary sclerosing cholangitis (PSC), a disorder of both intrahepatic and extrahepatic bile ducts (48). Implementation of endoscopic retrograde cholangiopancreatography in children has clarified that much of what was previously labeled as pericholangitis on histology represents small-duct PSC. Data from tertiary referral centers attracting the most affected patients suggest a range of PSC in $1.6 \%$ to $7.4 \%$ of patients with UC $(2,49,50)$. Olsson et al $(51)$, in a study of 1500 patients 16 years of age and older, reported that the prevalence of the hepatobiliary disease was $5.5 \%$ in patients with extensive colitis and $0.5 \%$ in patients with distal colitis. A marked male predominance is apparent in patients with colitis and cholangitis compared with those who have colitis without cholangitis.

The etiology of PSC is unknown. The biliary injury may be initiated by an immunemediated destruction of the hepatobiliary tract caused by transient infection, or the absorption of bacterial products in genetically predisposed individuals with colonic disease. Perinuclear antineutrophil cytoplasmic antibody titers are elevated in many patients with PSC and UC (52). Autoimmune disorders are more frequent in patients with PSC than in patients with IBD without liver disease; $25 \%$ of patients with PSC have at least 1 autoimmune disorder outside the liver and colon.

The diagnosis of PSC should be suspected in patients with elevated liver enzymes, specifically the alkaline phosphatase or $\gamma$-glutamyl transpeptidase levels. In advanced cases, the total and conjugated serum bilirubin levels are elevated. Serum immunoglobin-G is elevated, anti-smooth muscle and anti-nuclear antibodies are positive, and the HLA DR3, B8 haplotype is found in $70 \%$ of patients with PSC. Liver biopsies reveal ductular, portal, and peripheral inflammatory infiltrates, bile-duct proliferation, periductal fibrosis, and varying degrees of bridging fibrosis. 
Pharmacological therapies that have been evaluated in adult patients with PSC include prednisone, azathioprine, budesonide, methotrexate, cyclosporine, pentoxifylline, tacrolimus, bezafibrate, and antibiotics. To date, none of these treatments has been shown to affect the course of disease, including patient survival and eventual liver transplantation. Children with PSC experience significant improvements in their liver biochemical indices when treated with ursodeoxycholic acid, but their disease course remains unchanged (53). Transhepatic or endoscopic balloon dilatation and short-term stenting of strictures have provided clinical improvement of symptomatic strictures. Relief of strictures by surgical, endoscopic, or interventional radiological procedures for symptomatic strictures has prolonged survival time. Ponsioen et al (54) reported a 4-year experience with short-term treatment using polyethylene stents for dominant strictures in 32 adult patients with symptomatic PSC for a mean of 11 days. Cholestatic indices improved in $83 \%$ of patients at 2 months; $80 \%$ remained free of further endoscopic intervention for 1 year and $60 \%$ for 3 years. Surgical drainage procedures (eg, portoenterostomy, choledochoenterostomy) are ineffective, are associated with an increased risk of postoperative cholangitis, and may make subsequent liver transplantation technically more difficult. Orthotopic liver transplantation can successfully treat children with PSC, with excellent long-term patient and graft survival (55). Empiric oral vancomycin in children with PSC has shown some improvement in liver enzyme levels (56).

Nonalcoholic fatty liver disease (NAFLD) and nonalcoholic steatohepatitis (NASH) are relatively common and can slowly progress to end-stage liver disease. NAFLD sensitizes the liver to injury and increases the risk for developing acute or chronic liver failure following another liver injury. CD is a risk factor for developing NAFLD, which can evolve into NASH (57). In a study of Hungarian adult patients with IBD, Lakatos et al (2) found NASH in $9.4 \%$ of UC and $19.3 \%$ of patients with CD. Another investigation reported NAFLD in $39.5 \%$ of $\mathrm{CD}$ and $35.5 \%$ of patients with UC. The degree of hepatosteatosis was not related to the site, duration, and activity of the disease or the type of medication used (58).

Riegler et al (59) showed a significant association between hepatobiliary abnormalities in both disease duration and steroid and mesalamine treatment in UC patients. Monitoring of hepatobiliary function is important in IBD, even in the absence of symptoms.

Cholelithiasis is more likely a complication of the malabsorption in small intestinal disease and is reported in $11 \%$ of adults with CD (4). A recent case-control study in adults suggests that only patients with $\mathrm{CD}$ are at higher risk for gallstones compared with UC or matched controls (59 a). In a study of 583 patients with IBD, Stephano et al found that 56 (10.9\%) had cholethiasis, of which 41 (13.2\%) had CD and 15 (7.5\%) had UC. The mechanism of cholesterol-gallstone formation in $\mathrm{CD}$ may be related to an abnormal bile lipid composition after ileal resection or disease, and is likely a result of altered enterohepatic bilirubin circulation (60).

\section{IMPAIRED GROWTH}

Impaired growth, although also observed in UC, is more common in CD $(5,6,61)$. Low levels of insulin-like growth factor-1 are found in poorly nourished children with $\mathrm{CD}$ and growth delay; levels improve with nutritional restitution. In 184 children with IBD with EIMs, Stawarski et al (6) reported growth delay in $28 \%$ with UC and $80 \%$ with CD. Insulinlike growth factor-1 binding-protein levels are reported to be similar in growth impaired and normally growing children with $\mathrm{CD}$.

Chronic undernutrition is considered the primary pathogenic factor in growth impairment. Diminished oral intake (sometimes simply due to fear of exacerbation of gastrointestinal symptoms), nutrient malabsorption, or a protein-losing enteropathy can contribute to 
nutritional deficiencies. Some medications may contribute to under-nutrition. Chronic administration of high-dose daily corticosteroids therapy for as little as 7 to 14 days is associated with diminished type 1 collagen production, a prerequisite for linear growth. Alternate-day corticosteroid regimens appear to have less impact on growth velocity.

Medical therapy is not always successful in reducing disease activity to promote normal growth and development. Recent trials using growth hormone for children with $\mathrm{CD}$ show some benefit of this treatment (62). Surgical treatment may be required, and may significantly help children on high-dose corticosteroids with growth retardation. Surgery is curative in UC, allowing discontinuation of growth-suppressive therapies in this group of patients. Surgical intervention in CD, particularly if the disease is limited to a specific and relatively short segment of the intestine, has been shown to induce long-term remission and promote growth in prepubertal or early-pubertal children (63).

\section{EXTRAINTESTINAL MANIFESTATIONS SECONDARY TO COMPLICATION OF OR DIRECT EXTENSION OF BOWEL DISEASE}

\section{Genitourinary and Renal Manifestations}

Nephrolithiasis, obstructive uropathy, and fistulization of the urinary tract directly result from the underlying disease process. Reported incidence varies from $4 \%$ to $23 \%$ in adult patients but only in $1 \%$ to $2 \%$ of children with IBD (4).

Nephrolithiasis is due to calcium oxalate or uric acid stones. Calcium oxalate is due to hyperoxaluria typically associated with either distal ileal $\mathrm{CD}$ or ileal resection. Oxalate absorption increases in the presence of unabsorbed fatty acids in the colon. The fatty acids compete with oxalate to bind calcium, displacing the oxalate, which can then be absorbed as unbound sodium oxalate across colonocytes and excreted into the urine. Because sodium oxalate only is absorbed in the colon, calcium-oxalate stones form only in patients with an intact colon. Patients with an ileostomy are prone to formation of uric-acid stones because of frequent dehydration. The sudden onset of severe abdominal, back, or flank pain in patients with IBD, particularly if different from the usual discomfort, should lead to inclusion of a renal stone in the differential diagnosis.

The management of nephrolithiasis in patients with IBD is no different from that in the general population and consists of analgesia, hydration, alkalinization of urine for uric-acid stones, lithotripsy, and surgery. Patients with an ileostomy should be encouraged to drink adequate amounts of fluids.

Urological manifestations in patients with IBD may include ureteral calculi, enterovesical fistula, perivesical infection, perinephric abscess, and obstructive uropathy with hydronephrosis. Ureteral compression is associated with retroperitoneal extension of the phlegmonous inflammatory process involving the terminal ileum and cecum, and may result in hydronephrosis severe enough to cause hypertension. Surgical intervention usually is indicated for obstructive uropathy in patients with IBD. Fistulous disease complicating the urinary tract in patients with CD may present with pneumaturia or recurrent urinary tract infections. Surgical intervention may be required if medical therapy does not heal the fistula. Medical therapy includes azathioprine/6-mercaptopurine or anti-tumor necrosis factor- $\alpha$ agents, such as infliximab or thalidomide (64).

Immune complex glomerulonephritis presenting with proteinuria and hematuria has been described in children and adults with CD or UC (65). Diagnosis is by renal biopsy, and treatment parallels the underlying IBD. 
Secondary systemic amyloidosis is a rare but serious complication that has been reported in $0.9 \%$ and $0.07 \%$ of patients with CD and UC $(66,67)$, respectively. Amyloidosis in patients with IBD often affects the kidney and presents with proteinuria followed by nephritic syndrome and subsequent renal failure or death. Diagnosis is made with liver, rectal, or renal biopsy, although a fat pad aspiration is the most sensitive diagnostic test. Treatment includes colchicine and renal transplantation (68).

\section{Pancreatitis}

Pancreatitis may be associated with both UC and CD. The most common cause is iatrogenic and involves sensitivity to medications used to treat IBD (3\% of patients), including sulfasalazine, mesalamine, 6-mercaptopurine, and azathioprine. Pancreatitis may present as symptomatic (in $2 \%$ ) or more commonly asymptomatic (8-21\%) disease in adults with IBD. Le Large-Guiheneuf et al (69) reported pancreatitis in $27 \%$ of pediatric patients with IBD. In a study of 101 children, Stawarski et al (70) reported acute pancreatitis in $4.5 \%$ with CD and $5.1 \%$ with UC. Pancreatic auto antibodies have been found in up to $40 \%$ of patients with CD (71). Further studies are needed to demonstrate whether specific antibodies, apart from their diagnostic relevance for IBD, have a role in its pathogenesis and potentially identifying patients at risk. Diagnosis is confirmed by computed tomography with contrast, magnetic resonance imaging, or endoscopic retrograde cholangiopancreatography.

\section{Hematological Manifestations}

Although abnormalities involving all hematopoietic cell lines may be present, anemia is the most common hematological manifestation of IBD. Stawarski et al (6) reported secondary anemia in 5\% of patients with UC and 70\% with CD. Factors contributing to the development of the various forms of anemia include chronic gastrointestinal blood loss, inadequate dietary iron intake or absorption, vitamin $\mathrm{B}_{12}$ malabsorption secondary to terminal ileal disease or ileal resection, folate deficiency due to proximal small-bowel disease or sulfasalazine therapy, or autoimmune hemolysis. Leukocytosis and thrombocytopenia are usually due to immunosuppressant treatments or sulfasalazine. Plasma erythropoietin levels often are lower in patients with IBD than expected, in conjunction with severe anemia. Treatment of anemia depends on the underlying causes. Symptomatic anemia requires blood transfusion, whereas replacement of deficient vitamins may be required in other types. Recombinant erythropoietin has been successfully used in some patients with IBD and refractory anemia.

Thrombocytosis and thromboembolic events resulting from a hypercoagulable state in patients with IBD can lead to pulmonary embolism or thrombosis elsewhere in the body. Thrombosis has been reported in $1.8 \%$ of patients with UC and $3.1 \%$ of patients with CD. Thromboembolism and thrombosis are less frequently reported among pediatric patients, with 3 patients with UC and 1 with CD described in case reports (72). Factors such as inflammation, fluid depletion, immobilization, surgery, steroid therapy, and use of central venous catheters are prothrombic risk factors in patients with IBD. The development of thromboses may be related to thrombocytosis and an increase in acute-phase reactants during active inflammation. Inflammatory mediators and hypercoagulation are involved in the pathogenesis of IBD, and some proinflammatory cytokines such as interleukin-6 are thrombogenic (73-75). A population-based study in adults suggests that IBD patients have 3 -fold greater risk compared with subjects without IBD for developing deep venous thrombosis and pulmonary embolism (76). Factor V Leiden mutation is the most frequent cause of inherited thrombophilia, but studies have shown no difference in the prevalence of Factor V Leiden between patients with IBD and healthy controls. Prevention includes adequate control of disease activity and vitamin supplementation. Medications that affect clotting factors such as warfarin, heparin, and low-molecular-weight heparin have been used 
in life-threatening hemorrhage. The risk of intestinal bleeding should always be considered with the use of these medications.

\section{Cardiovascular Manifestations}

Children and adults with IBD have been rarely $(<1 \%)$ reported to develop pleuropericarditis either at initial presentation or during active or quiescent disease $(77,78)$. The pathogenesis of pleuropericarditis is unknown, although certain medications (eg, sulfasalazine and mesalamine derivatives) have been implicated in some cases $(79,80)$. The clinical presentation may include chest pain, dyspnea, or in severe cases pericardial tamponade requiring rapid drainage. Nonsteroidal anti-inflammatory drugs have been used as therapy, although this should be weighed against the hypothetical risk of exacerbating the underlying IBD.

\section{Pulmonary Manifestations}

Deterioration of pulmonary function appears to parallel underlying disease activity in patients with IBD. Sulfasalazine-associated lung disease, although rare, is observed after at least 2 months of therapy and includes eosinophilic pneumonia, fibrosing alveolitis, and interstitial pneumonitis. Other pulmonary disorders that have been associated with IBD include pulmonary vasculitis, apical fibrosis, bronchiectasis, bronchitis, bronchiolitis, and granulomatous lung disease. These pulmonary pathological processes do not typically relate to the severity or activity of the underlying IBD. Tuberculosis and other opportunistic infections should be considered in patients taking immunosuppressive medications.

\section{Neurological Manifestations}

Central and peripheral neurological disorders are described in patients with IBD and include peripheral neuropathies, myopathies, focal central nervous system defects, convulsions, confusional episodes, meningitis, syncope, optic neuritis, and sensorineural loss (81). Autoimmune mechanisms are proposed for involvement with IBD. Nutritional deficiencies associated with neurological manifestations, such as vitamin $\mathrm{B}_{12}$ deficiency, should be investigated. Spinal abscess has been reported in both a child and an adult with initial complaints of severe back pain due to extension of a psoas abscess from the epidural space to the subarachnoid space (82). Careful neurological examination is important in patients developing psoas abscess secondary to $\mathrm{CD}$.

Medications for treatment of IBD also are associated with neurological defects. A greater risk for development of a "stocking glove" peripheral neuropathy is observed with chronic and higher dose metronidazole therapy (83). Although about 50\% of patients treated with metronidazole (mean dose, $15-20 \mathrm{mg} \cdot \mathrm{kg}^{-1} \cdot \mathrm{day}^{-1}$ ) for $\leq 6$ months develop paresthesias, $>80 \%$ will have a sensory peripheral neuropathy demonstrated on nerve conduction-velocity testing. Symptoms may persist even after discontinuation of the medication.

\section{SUMMARY}

UC and CD commonly manifest signs and symptoms of extraintestinal disease. Patients with evidence of disease extrinsic to the gastrointestinal tract should be considered to have an associated complication of their underlying IBD. Patients with persistent signs or symptoms of EIMs should be evaluated for IBD if not previously diagnosed. Data on EIMs of IBD in children are lacking compared with those in adults (Table 2), and may be revealing in light of recent studies suggesting unique aspects of early-onset IBD compared with older patients $(91,92)$. Information pertaining to the incidence and prevalence of EIMs of IBD in children is essential to improve our understanding of the natural history and associated EIMs in children with IBD. 


\section{Acknowledgments}

This work is supported in part by NIH grants T32 DK007762 and K24 DK060617.

\section{References}

1. Bernstein CN, Blanchard JF, Rawsthorne P, et al. The prevalence of extraintestinal diseases in inflammatory bowel disease: a population-based study. Am J Gastroenterol. 2001; 96:1116-22. [PubMed: 11316157]

2. Lakatos L, Pandur T, David G, et al. Association of extraintestinal manifestations of inflammatory bowel disease in a province of western Hungary with disease phenotype: results of a 25-year follow-up study. World J Gastroenterol. 2003; 9:2300-7. [PubMed: 14562397]

3. Mendoza JL, Lana R, Taxonera C, et al. Extraintestinal manifestations in inflammatory bowel disease: differences between Crohn's disease and ulcerative colitis. Med Clin (Barc). 2005; 125:297-300. [PubMed: 16159555]

4. Repiso A, Alcantara M, Munoz-Rosas C, et al. Extraintestinal manifestations of Crohn's disease: prevalence and related factors. Rev Esp Enferm Dig. 2006; 98:510-7. [PubMed: 17022700]

5. Grossman BJ, DeBenedetti CD. Extraintestinal manifestations of chronic inflammatory bowel disease in children. Proc Inst Med Chic. 1970; 28:119. [PubMed: 5430559]

6. Stawarski A, Iwanczak B, Krzesiek E, et al. Intestinal complications and extraintestinal manifestations in children with inflammatory bowel disease. Pol Merkur Lekarski. 2006; 20:22-5. [PubMed: 16617729]

7. Greenstein AJ, Janowitz HD, Sachar DB. The extra-intestinal complications of Crohn's disease and ulcerative colitis: a study of 700 patients. Medicine (Baltimore). 1976; 55:401-12. [PubMed: 957999]

8. Snook JA, de Silva HJ, Jewell DP. The association of autoimmune disorders with inflammatory bowel disease. Q J Med. 1989; 72:835-40. [PubMed: 2616728]

9. Rath HC, Herfarth HH, Ikeda JS, et al. Normal luminal bacteria, especially Bacteroides species, mediate chronic colitis, gastritis, and arthritis in HLA-B27/human beta2 microglobulin transgenic rats. J Clin Invest. 1996; 98:945-53. [PubMed: 8770866]

10. Nguyen GC, Torres EA, Regueiro M, et al. Inflammatory bowel disease characteristics among African Americans, Hispanics, and non-Hispanic whites: characterization of a large North American cohort. Am J Gastroenterol. 2006; 101:1012-23. [PubMed: 16696785]

11. Roussomoustakaki M, Satsangi J, Welsh K, et al. Genetic markers may predict disease behavior in patients with ulcerative colitis. Gastroenterology. 1997; 112:1845-53. [PubMed: 9178675]

12. Orchard TR, Chua CN, Ahmad T, et al. Uveitis and erythema nodosum in inflammatory bowel disease: clinical features and the role of HLA genes. Gastroenterology. 2002; 123:714-8. [PubMed: 12198697]

13. Lindgren A, Wallerstedt S, Olsson R. Prevalence of Crohn's disease and simultaneous occurrence of extraintestinal complications and cancer. An epidemiologic study in adults. Scand J Gastroenterol. 1996; 31:74-8. [PubMed: 8927944]

14. Mir-Madjlessi SH, Taylor JS, Farmer RG. Clinical course and evolution of erythema nodosum and pyoderma gangrenosum in chronic ulcerative colitis: a study of 42 patients. Am J Gastroenterol. 1985; 80:615-20. [PubMed: 4025277]

15. Monsen U, Sorstad J, Hellers G, et al. Extracolonic diagnoses in ulcerative colitis: an epidemiological study. Am J Gastroenterol. 1990; 85:711-6. [PubMed: 2353691]

16. Gellert A, Green ES, Beck ER, et al. Erythema nodosum progressing to pyoderma gangrenosum as a complication of Crohn's disease. Postgrad Med J. 1983; 59:791-3. [PubMed: 6657543]

17. Powell RJ, Holbrook MR, Stevens A. Pyoderma gangrenosum and its treatment. Lancet. 1997; 350:1720-1. [PubMed: 9413459]

18. Barrie A, Plevy S. Treatment of immune-mediated extraintestinal manifestations of inflammatory bowel disease with infliximab. Gastroenterol Clin North Am. 2006; 35:883-93. [PubMed: 17129819] 
19. Kugathasan S, Miranda A, Nocton J, et al. Dermatologic manifestations of Crohn disease in children: response to infliximab. J Pediatr Gastroenterol Nutr. 2003; 37:150-4. [PubMed: 12883301]

20. Yu JT, Chong LY, Lee KC. Metastatic Crohn's disease in a Chinese girl. Hong Kong Med J. 2006; 12:467-9. [PubMed: 17148802]

21. Ytting H, Vind I, Bang D, et al. Sweet's syndrome-an extra-intestinal manifestation in inflammatory bowel disease. Digestion. 2005; 72:195-200. [PubMed: 16179788]

22. Orchard TR, Thiyagaraja S, Welsh KI, et al. Clinical phenotype is related to HLA genotype in the peripheral arthropathies of inflammatory bowel disease. Gastroenterology. 2000; 118:274-8. [PubMed: 10648455]

23. Banet DE, McClave SA, Callen JP. Oral metronidazole, an effective treatment for Sweet's syndrome in a patient with associated inflammatory bowel disease. J Rheumatol. 1994; 21:1766-8. [PubMed: 7799365]

24. Kemmett D, Hunter JA. Sweet's syndrome: a clinicopathologic review of twenty-nine cases. J Am Acad Dermatol. 1990; 23:503-7. [PubMed: 2212153]

25. Buahene K, Hudson M, Mowat A, et al. Erythema elevatum diutinum — an unusual association with ulcerative colitis. Clin Exp Dermatol. 1991; 16:204-6. [PubMed: 1934575]

26. Elsner J, Kiehl P, Kapp A, et al. Erythema elevatum and diutinum in Crohn disease. Hautarzt. 1996; 47:701-4. [PubMed: 8999026]

27. Rankin GB. Extraintestinal and systemic manifestations of inflammatory bowel disease. Med Clin North Am. 1990; 74:39-50. [PubMed: 2404180]

28. Veloso FT, Carvalho J, Magro F. Immune-related systemic manifestations of inflammatory bowel disease. A prospective study of 792 patients. J Clin Gastroenterol. 1996; 23:29-34. [PubMed: 8835896]

29. Caspi RR, Stiff LR, Morawetz R, et al. Cytokine-dependent modulation of oral tolerance in a murine model of autoimmune uveitis. Ann N Y Acad Sci. 1996; 778:315-24. [PubMed: 8610985]

30. Yang P, de Vos AF, Kijlstra A. Macrophages and MHC class II positive cells in the choroid during endotoxin induced uveitis. Br J Ophthalmol. 1997; 81:396-401. [PubMed: 9227206]

31. Tripathi RC, Kirschner BS, Kipp M, et al. Corticosteroid treatment for inflammatory bowel disease in pediatric patients increases intraocular pressure. Gastroenterology. 1992; 102:1957-61. [PubMed: 1587414]

32. Protzer U, Duchmann R, Hohler T, et al. Enteropathic spondylarthritis in chronic inflammatory bowel diseases: prevalence, manifestation pattern and HLA association. Med Klin (Munich). 1996; 91:330-5. [PubMed: 8767304]

33. Passo MH, Fitzgerald JF, Brandt KD. Arthritis associated with inflammatory bowel disease in children. Relationship of joint disease to activity and severity of bowel lesion. Dig Dis Sci. 1986; 31:492-7. [PubMed: 3698765]

34. Nunez C, Alecsandru DM, Mendoza JL, et al. Genetic markers linked to rheumatoid arthritis are also strongly associated with articular manifestations in ulcerative colitis patients. Hum Immunol. 2006; 67:324-30. [PubMed: 16720213]

35. Miner PB Jr. Factors influencing the relapse of patients with inflammatory bowel disease. Am J Gastroenterol. 1997; 92:1S-4S. [PubMed: 9395345]

36. Keyser FD, Mielants H, Veys EM. Current use of biologicals for the treatment of spondyloarthropathies. Expert Opin Pharmacother. 2001; 2:85-93. [PubMed: 11336571]

37. Bernstein CN. Osteoporosis and other complications of inflammatory bowel disease. Curr Opin Gastroenterol. 2002; 18:428-34. [PubMed: 17033317]

38. Motley RJ, Clements D, Evans WD, et al. A four-year longitudinal study of bone loss in patients with inflammatory bowel disease. Bone Miner. 1993; 23:95-104. [PubMed: 8305881]

39. Szumera M, Landowski P, Kaminska B, et al. Bone mineral density in inflammatory bowel diseases in children. Med Wieku Rozwoj. 2006; 10:445-51. [PubMed: 16825715]

40. Walther F, Fusch C, Radke M, et al. Osteoporosis in pediatric patients suffering from chronic inflammatory bowel disease with and without steroid treatment. J Pediatr Gastroenterol Nutr. 2006; 43:42-51. [PubMed: 16819376] 
41. Vestergaard P. Prevalence and pathogenesis of osteoporosis in patients with inflammatory bowel disease. Minerva Med. 2004; 95:469-80. [PubMed: 15785432]

42. Ross PD. Osteoporosis. Frequency, consequences, and risk factors. Arch Intern Med. 1996; 156:1399-411. [PubMed: 8678708]

43. Black DM, Cummings SR, Karpf DB, et al. Randomised trial of effect of alendronate on risk of fracture in women with existing vertebral fractures. Fracture Intervention Trial Research Group. Lancet. 1996; 348:1535-41. [PubMed: 8950879]

44. Vakil N, Sparberg M. Steroid-related osteonecrosis in inflammatory bowel disease. Gastroenterology. 1989; 96:62-7. [PubMed: 2909438]

45. Marmor L. Unicompartmental arthroplasty for osteonecrosis of the knee joint. Clin Orthop Relat Res. 1993; 294:247-53. [PubMed: 8358924]

46. Ficarra G, Cicchi P, Amorosi A, et al. Oral Crohn's disease and pyostomatitis vegetans. An unusual association. Oral Surg Oral Med Oral Pathol. 1993; 75:220-4. [PubMed: 8426722]

47. Philpot HC, Elewski BE, Banwell JG, et al. Pyostomatitis vegetans and primary sclerosing cholangitis: markers of inflammatory bowel disease. Gastroenterology. 1992; 103:668-74. [PubMed: 1634082]

48. Wee A, Ludwig J. Pericholangitis in chronic ulcerative colitis: primary sclerosing cholangitis of the small bile ducts? Ann Intern Med. 1985; 102:581-7. [PubMed: 3985511]

49. Bernstein CN. Extraintestinal manifestations of inflammatory bowel disease. Curr Gastroenterol Rep. 2001; 3:477-83. [PubMed: 11696285]

50. Rasmussen HH, Fallingborg J, Mortensen PB, et al. Primary sclerosing cholangitis in patients with ulcerative colitis. Scand J Gastroenterol. 1992; 27:732-6. [PubMed: 1411277]

51. Olsson R, Danielsson A, Jarnerot G, et al. Prevalence of primary sclerosing cholangitis in patients with ulcerative colitis. Gastroenterology. 1991; 100:1319-23. [PubMed: 2013375]

52. Duerr RH, Targan SR, Landers CJ, et al. Neutrophil cytoplasmic antibodies: a link between primary sclerosing cholangitis and ulcerative colitis. Gastroenterology. 1991; 100:1385-91. [PubMed: 2013383]

53. Lindor KD. Ursodiol for primary sclerosing cholangitis. Mayo Primary Sclerosing CholangitisUrsodeoxycholic Acid Study Group. N Engl J Med. 1997; 336:691-5. [PubMed: 9041099]

54. Ponsioen CY, Lam K, van Milligen de Wit AW, et al. Four years experience with short term stenting in primary sclerosing cholangitis. Am J Gastroenterol. 1999; 94:2403-7. [PubMed: 10483999]

55. Liden H, Norrby J, Gabel M, et al. Outcome after liver transplantation for primary sclerosing cholangitis. Transplant Proc. 2001; 33:2452-3. [PubMed: 11406207]

56. Cox KL, Cox KM. Oral vancomycin: treatment of primary sclerosing cholangitis in children with inflammatory bowel disease. J Pediatr Gastroenterol Nutr. 1998; 27:580-3. [PubMed: 9822326]

57. Canbay A, Bechmann LP, Best J, et al. Crohn's disease-induced non-alcoholic fatty liver disease (NAFLD) sensitizes for severe acute hepatitis B infection and liver failure. Z Gastroenterol. 2006; 44:245-8. [PubMed: 16514570]

58. Bargiggia S, Maconi G, Elli M, et al. Sonographic prevalence of liver steatosis and biliary tract stones in patients with inflammatory bowel disease: study of 511 subjects at a single center. J Clin Gastroenterol. 2003; 36:417-20. [PubMed: 12702985]

59. Riegler G, D'Inca R, Sturniolo GC, et al. Hepatobiliary alterations in patients with inflammatory bowel disease: a multicenter study. Caprilli \& Gruppo Italiano Studio Colon-Retto. Scand J Gastroenterol. 1998; 33:93-98. [PubMed: 9489915]

59a. Parente F, Pastore L, Bargiggia S, et al. Incidence and risk factors for gallstones in patients with inflammatory bowel disease: a large case-control study. Hepatology. 2007; 45:1267-74. [PubMed: 17464998]

60. Brink MA, Slors JF, Keulemans YC, et al. Enterohepatic cycling of bilirubin: a putative mechanism for pigment gallstone formation in ileal Crohn's disease. Gastroenterology. 1999; 116:1420-7. [PubMed: 10348826]

61. Motil KJ, Grand RJ, Davis-Kraft L, et al. Growth failure in children with inflammatory bowel disease: a prospective study. Gastroenterology. 1993; 105:681-91. [PubMed: 8359640] 
62. Heyman MB, Wojcicki J, Kaplan S. Growth hormone in pediatric Crohn's disease. J Pediatr Gastroenterol Nutr. 2005; 41:556-7.

63. Singh Ranger G, Lamparelli MJ, Aldridge A, et al. Surgery results in significant improvement in growth in children with Crohn's disease refractory to medical therapy. Pediatr Surg Int. 2006; 22:347-52. [PubMed: 16518590]

64. Stein RB, Lichtenstein GR. Medical therapy for Crohn's disease: the state of the art. Surg Clin North Am. 2001; 81:71-101. [PubMed: 11218170]

65. Wilcox GM, Aretz HT, Roy MA, et al. Glomerulonephritis associated with inflammatory bowel disease. Report of a patient with chronic ulcerative colitis, sclerosing cholangitis, and acute glomerulonephritis. Gastroenterology. 1990; 98:786-91. [PubMed: 2137104]

66. Gitkind MJ, Wright SC. Amyloidosis complicating inflammatory bowel disease. A case report and review of the literature. Dig Dis Sci. 1990; 35:906-8. [PubMed: 2194769]

67. Greenstein AJ, Sachar DB, Panday AK, et al. Amyloidosis and inflammatory bowel disease. A 50year experience with 25 patients. Medicine (Baltimore). 1992; 71:261-70. [PubMed: 1522802]

68. Lovat LB, Madhoo S, Pepys MB, et al. Long-term survival in systemic amyloid A amyloidosis complicating Crohn's disease. Gastroenterology. 1997; 112:1362-5. [PubMed: 9098022]

69. Le Large-Guiheneuf C, Hugot JP, Faure C, et al. Pancreatic involvement in inflammatory bowel diseases in children. Arch Pediatr. 2002; 9:469-77. [PubMed: 12053540]

70. Stawarski A, Iwanczak F. Incidence of acute pancreatitis in children with inflammatory bowel disease. Pol Merkur Lekarski. 2004; 17:33-6. [PubMed: 15559607]

71. Stocker W, Otte M, Ulrich S, et al. Autoimmunity to pancreatic juice in Crohn's disease. Results of an autoantibody screening in patients with chronic inflammatory bowel disease. Scand J Gastroenterol Suppl. 1987; 139:41-52. [PubMed: 3324299]

72. Paradis K, Bernstein ML, Adelson JW. Thrombosis as a complication of inflammatory bowel disease in children: a report of four cases. J Pediatr Gastroenterol Nutr. 1985; 4:659-62. [PubMed: 4032181]

73. Gabay C, Kushner I. Acute-phase proteins and other systemic responses to inflammation. N Engl J Med. 1999; 340:448-54. [PubMed: 9971870]

74. McEver RP. Adhesive interactions of leukocytes, platelets, and the vessel wall during hemostasis and inflammation. Thromb Haemost. 2001; 86:746-56. [PubMed: 11583304]

75. Stouthard JM, Levi M, Hack CE, et al. Interleukin-6 stimulates coagulation, not fibrinolysis, in humans. Thromb Haemost. 1996; 76:738-42. [PubMed: 8950783]

76. Bernstein CN, Blanchard JF, Houston DS, et al. The incidence of deep venous thrombosis and pulmonary embolism among patients with inflammatory bowel disease: a population-based cohort study. Thromb Haemost. 2001; 85:430-4. [PubMed: 11307809]

77. Frid C, Bjarke B, Eriksson M. Myocarditis in children with inflammatory bowel disease. J Pediatr Gastroenterol Nutr. 1986; 5:964-5. [PubMed: 3794918]

78. Patwardhan RV, Heilpern RJ, Brewster AC, et al. Pleuropericarditis: an extraintestinal complication of inflammatory bowel disease. Report of three cases and review of literature. Arch Intern Med. 1983; 143:94-6. [PubMed: 6849612]

79. Agnholt J, Sorensen HT, Rasmussen SN, et al. Cardiac hypersensitivity to 5-aminosalicylic acid. Lancet. 1989; 1:1135. [PubMed: 2566070]

80. Gujral N, Friedenberg F, Friedenberg J, et al. Pleuropericarditis related to the use of mesalamine. Dig Dis Sci. 1996; 41:624-6. [PubMed: 8617147]

81. Sullivan SN. Sulfasalazine lung. Desensitization to sulfasalazine and treatment with acrylic coated 5-ASA and azodisalicylate. J Clin Gastroenterol. 1987; 9:461-3. [PubMed: 2888803]

82. Hefter H, Piontek M, Aulich A. Bacterial meningitis and dorsal spinal epidural abscess caused by Crohn's disease. Neurology. 1991; 41:606-8. [PubMed: 2011270]

83. Duffy LF, Daum F, Fisher SE, et al. Peripheral neuropathy in Crohn's disease patients treated with metronidazole. Gastroenterology. 1985; 88:681-4. [PubMed: 2981752]

84. Orchard T. Extraintestinal complications of inflammatory bowel disease. Curr Gastroenterol Rep. 2003; 5:512-7. [PubMed: 14602062] 
85. Al-Ratrout JT, Ansari NA. Epidermolysis bullosa acquisita and Crohn's disease. Saudi Med J. 2004; 25:1736-8. [PubMed: 15573217]

86. Gluck M, Kayne A. Acquired epidermolysis bullosa and Crohn's disease. Gastrointest Endosc. 2003; 57:563-4. [PubMed: 12665771]

87. Benton EC, Rutherford D, Hunter JA. Sweet's syndrome and pyoderma gangrenosum associated with ulcerative colitis. Acta Derm Venereol. 1985; 65:77-80. [PubMed: 2578713]

88. Muller L, Bala G, Kuczynska R, et al. Pyoderma gangrenosum, a nonintestinal manifestation of Lesniowski-Crohn's disease in a 15 years old girl. Med Wieku Rozwoj. 2006; 10:463-73. [PubMed: 16825717]

89. Clark JH, Fitzgerald JF, Bergstein JM. Nephrolithiasis in childhood inflammatory bowel disease. J Pediatr Gastroenterol Nutr. 1985; 4:829-34. [PubMed: 2995632]

90. Kahn E, Markowitz J, Simpser E, et al. Amyloidosis in children with inflammatory bowel disease. J Pediatr Gastroenterol Nutr. 1989; 8:447-53. [PubMed: 2723936]

91. Heyman MB, Kirschner BS, Gold BD, et al. Children with early-onset inflammatory bowel disease (IBD): analysis of a pediatric IBD consortium registry. J Pediatr. 2005; 146:35-40. [PubMed: 15644819]

92. Gupta N, Cohen SA, Bostrom AG, et al. Risk factors for initial surgery in pediatric patients with Crohn's disease. Gastroenterology. 2006; 130:1069-77. [PubMed: 16618401] 
TABLE 1

Extraintestinal manifestation of inflammatory bowel disease

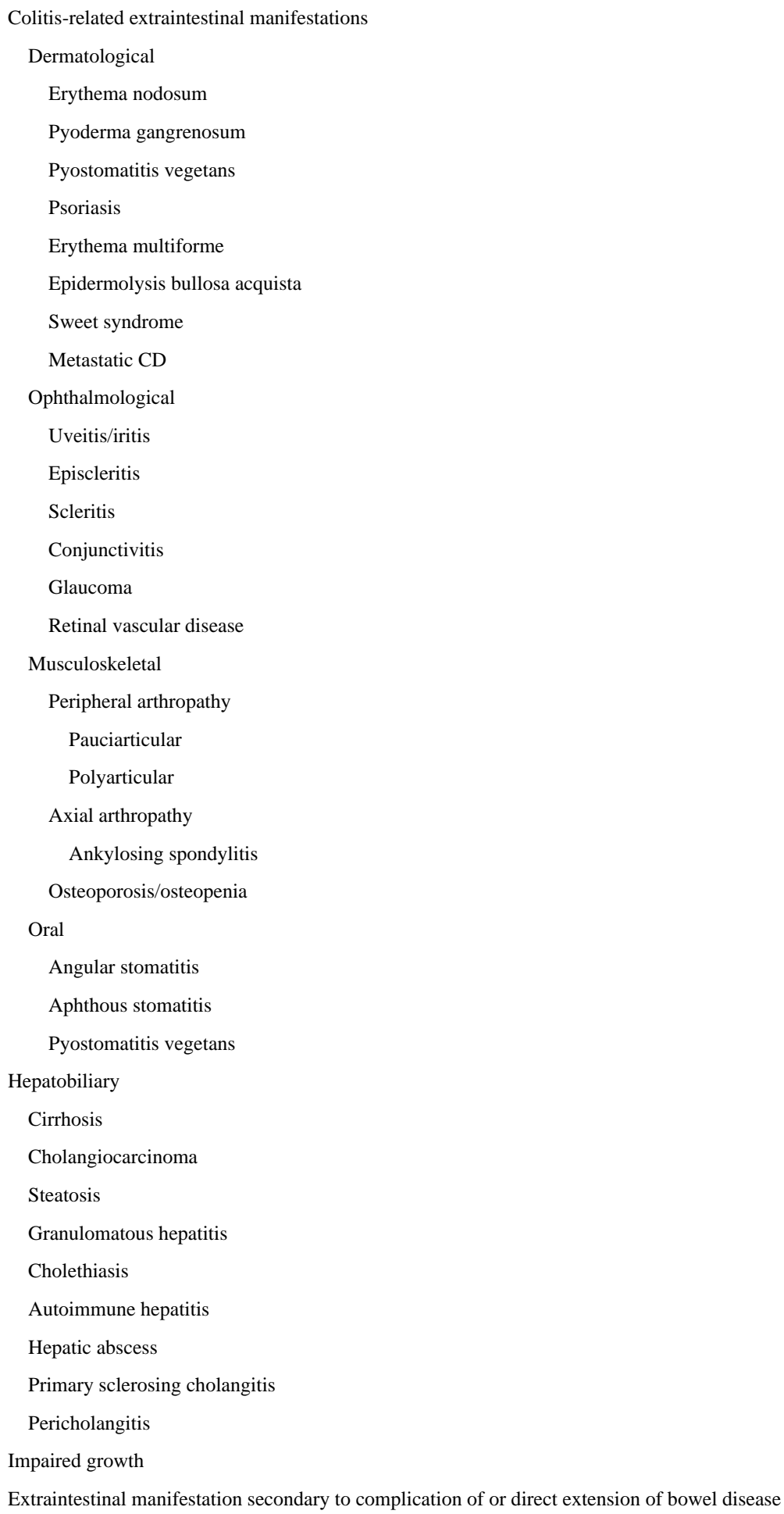

Extraintestinal manifestation secondary to complication of or direct extension of bowel disease 


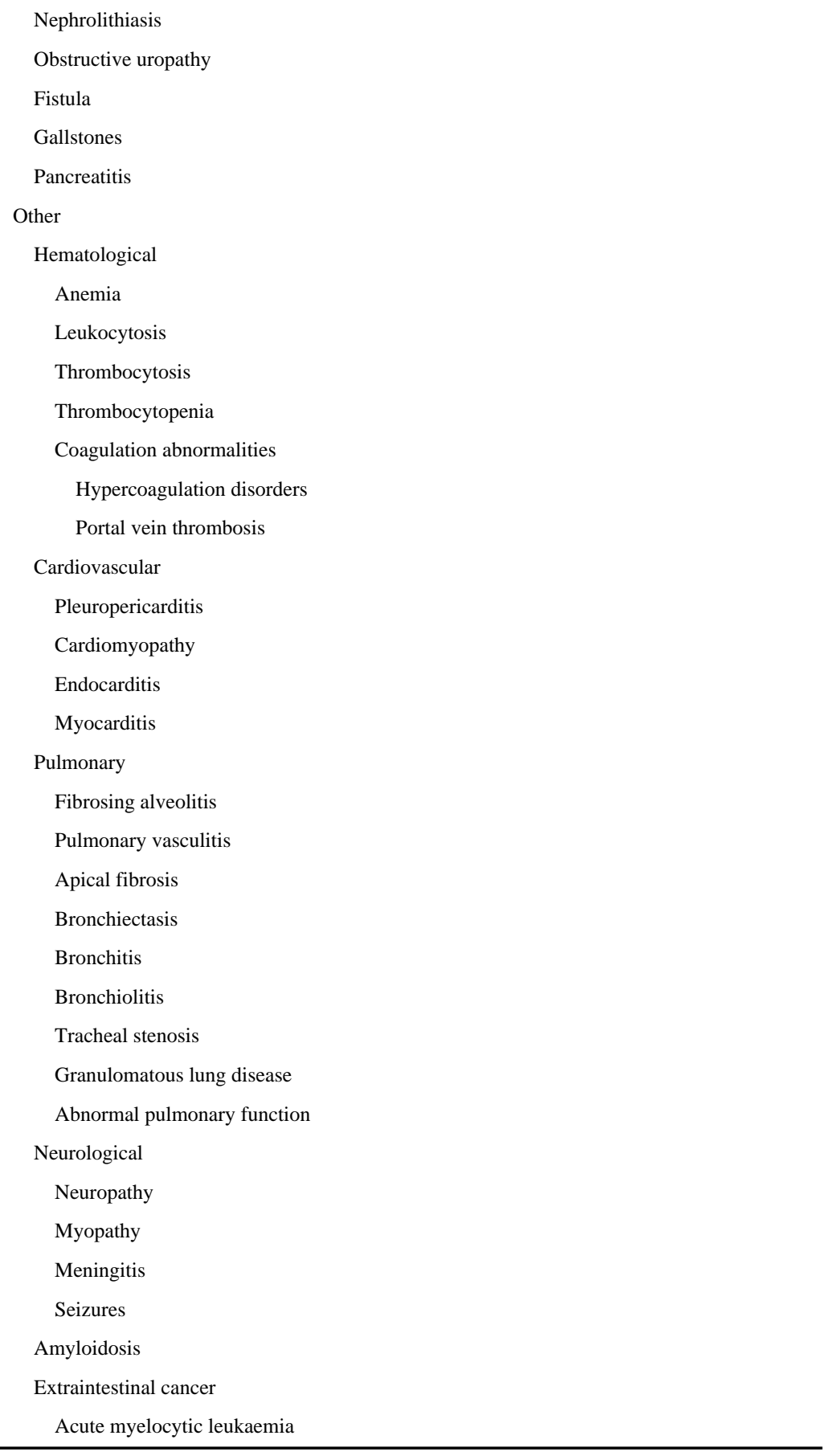


TABLE 2

Prevalence of extraintestinal manifestations of inflammatory bowel disease

\begin{tabular}{|c|c|c|}
\hline \multirow[b]{2}{*}{ Extraintestinal manifestation } & \multicolumn{2}{|c|}{ Prevalence in adults with IBD } \\
\hline & UC EIM (\%) & CD EIM (\%) \\
\hline Dermatology $(2,7)$ & $3.8-19$ & $10.2-23$ \\
\hline Erythema nodosum $(2,7,13-15,84)$ & $0.9-4$ & $0.7-15$ \\
\hline Pyoderma gangrenosum $(2,7)$ & $0.5-5$ & $0.8-1.6$ \\
\hline Epidermolysis bullosa $(85,86)$ & & $\mathrm{CR}$ \\
\hline Sweet syndrome $(21,87)$ & $\mathrm{CR}$ & \\
\hline Uveitis/iritis $(2,7)$ & $1.5-4$ & $2-13$ \\
\hline Joints/arthritis $(2,7)$ & $10.2-26$ & $22.4-39$ \\
\hline Ankylosing spondylitis $(2,7)$ & $1.5-9$ & $2.7-17$ \\
\hline Osteoporosis and osteopenia (7) & 9 & 17 \\
\hline $\operatorname{PSC}(1,2)$ & $3-12.4$ & $0.4-22.4$ \\
\hline NAFLD/NASH (2) & 9.4 & 19.3 \\
\hline Nephrolithiasis (7) & 5 & 5 \\
\hline Amyloidosis (67) & 0.7 & 0.9 \\
\hline Pancreatitis (7) & $0.2-0.5$ & 0.4 \\
\hline Anemia (2) & 17.7 & 9.6 \\
\hline Vascular thrombosis (2) & 1.8 & \\
\hline Oral aphthous ulcers $(2,7)$ & $0.5-4$ & 11 \\
\hline \multirow[t]{2}{*}{ Extraintestinal manifestation } & \multicolumn{2}{|c|}{$\underline{\text { Prevalence in children with IBD }}$} \\
\hline & UC EIM (\%) & CD EIM $(\%)$ \\
\hline Pyoderma gangrenosum (88) & $\mathrm{CR}^{*}$ & \\
\hline Joints/arthritis (6) & 3.8 & - \\
\hline Osteoporosis and osteopenia $(6,39)$ & $24-25$ & $8-41$ \\
\hline Growth delay (6) & 28 & 81 \\
\hline Nephrolithiasis $(6,89)$ & 3.2 & $\mathrm{CR}$ \\
\hline Autoimmune hepatitis (6) & 1.3 & - \\
\hline Uveitis (6) & 0.63 & - \\
\hline Amyloidosis (90) & $\mathrm{CR}$ & \\
\hline Vascular damage (6) & 0.63 & 4.2 \\
\hline Pancreatitis (6) & 2.5 & 3.9 \\
\hline Anemia (6) & 40.5 & 69 \\
\hline
\end{tabular}

